\title{
A Note on Prime Square Dominating Graph
}

\author{
A. Sudhakaraiah ${ }^{1}$, B. Narayana ${ }^{2}$ \\ ${ }^{1}$ Department of Mathematics, Sri Venkateswara University, Tirupathi, \\ ${ }^{2}$ Department of Basic Science and Humanities, St. Mary's Women's Engineering College, Guntur, A.P.India.
}

\begin{abstract}
In this paper we study about the prime square dominating graph and we observe that a prime dominating graph need not be prime square dominating graph, but every prime square dominating graph is prime dominating graph. In this paper we got interesting characteristics theorems such as if a graph contains an odd cycle then it is not a prime square dominating graph, if graph is bipartite then it is a prime square dominating graph. Moreover we discussed about minimal prime square dominating graph and provide necessary examples.
\end{abstract}

Keywords: Dominating graph, Prime dominating graph, Prime Square Dominating graph, minimal dominating, minimal prime square dominating graphs.

\section{Introduction}

Graph Theory is one of the most beautiful branch in branches of modern mathematics and Computer Sciences which has a wide range of research scope. For last 30 years the growth of graph theory has a many applications to describe combinatorial problems and algebraic problems. Graph Theory is the subject that which can be useful to understand the real life situations in better manner. One of the important studies in graph theory is about the graph labeling. In this section we collected necessary definitions, observations and some useful results from the literature that are useful for next section. For further basic definitions and examples we refer Satyanarayana, Syam Prasad [4], S.R Sudarshan Iyengar, Somindu C.R. and Swathi Srivatsan[5], Harary [1].

1.1 Definition: A linear graph (or simply a graph) $\mathrm{G}=(\mathrm{V}, \mathrm{E})$ consists of a set of objects $\mathrm{V}=\left\{\mathrm{v}_{1}, \mathrm{v}_{2}, \ldots,\right\}$ called vertices, and another set $E=\left\{\mathrm{e}_{1}, \mathrm{e}_{2}, \ldots\right\}$, whose elements are called edges, such that each edge $\mathrm{e}_{\mathrm{k}}$ is identified with an unordered pair $\left(\mathrm{v}_{\mathrm{i}}, \mathrm{v}_{\mathrm{j}}\right)$ of vertices. $\mathrm{v}_{\mathrm{i}}$, and $\mathrm{v}_{\mathrm{j}}$ are called the end points of $\mathrm{e}_{\mathrm{k}}$. If $\mathrm{V}, \mathrm{E}$ are finite sets, then the graph is called a finite graph.

1.2 Definitions: (i) Let $u$ and $v$ be two vertices of a graph $G$. We say that $u$ and $v$ are adjacent if there is an edge between $\mathrm{u}$ and $\mathrm{v}$.

(ii) An edge $\overline{\mathrm{VV}}$ for any $\mathrm{v} \in \mathrm{V}$ is called a loop (or self loop).

(iii) Two edges e and $f$ in a graph $G=(V, E)$ is said to be multiple edges (or parallel edges) if $e \neq f$ and the end vertices associated with e and $f$ are same.

(iv) A graph $\mathrm{G}$ is said to be simple if it contains no loops and multiple edges.

1.3 Definition: A walk is defined as a finite alternating sequence of vertices and edges, (no repetition of edge allowed) beginning and ending with vertices, such that each edge is incident with the vertices preceding and following it.

1.4 Definition: A walk is said to be a closed walk if the terminal points are same. A closed walk in which no vertex (except the initial vertex and final vertex) appears more than once is called a circuit or cycle.

1.5 Note: If cycle contains an odd number of edges then we say it is an odd cycle, if cycle contains an even number of edges then we say it is an even cycle.

1.6 Definition: If the vertices $v_{0}, v_{1}, \ldots, v_{k}$ of the walk $v_{0} e_{1} v_{1} e_{2} v_{2} \ldots e_{k} v_{k}$ are distinct then, the walk is called a path.

1.7 Definition: A graph $G$ is said to be connected if there is at least one path between every pair of vertices in G. A graph is said to be disconnected if it is not connected.

1.8 Definition: A graph $\mathrm{G}$ is said to be a bipartite graph if the vertex set can be partitioned in to two sets such that every edge of $\mathrm{G}$ having one end in one vertex set and another in other vertex set. 
1.9 Definition: In a graph $\mathrm{G}$, a subset $\mathrm{S}$ of $\mathrm{V}(\mathrm{G})$ is said to be a dominating set if every vertex not in $\mathrm{S}$ has a neighbor in $\mathrm{S}$. The domination number, denoted by $\gamma(\mathrm{G})$ as $\min \{|\mathrm{S}| / \mathrm{S}$ is a dominating set in $\mathrm{G}\}$.

1.10 Definition: Labeling of a graph plays a vital role in graph theory. A labeling of a graph $G$ is an assignment of distinct positive integers to its vertices. That is, an injective function $\mathrm{f}: \mathrm{V}(\mathrm{G}) \rightarrow \mathrm{N}$. Where, $\mathrm{N}$ denotes the set of natural numbers.

1.11 Definition: A graph $G(V, E)$ is said to be prime dominating graph, if for any two vertices $x, y$ in the vertex set $V$ of graph $G$ with $f(x)>f(y)$, are adjacent if $f(y)$ divides $f(x)$ and $f(y)$ is prime number.

In this paper we consider a kind of labeling in which there exists no rule that assigns values to edges. The vertex labeling is chosen from the set of positive integers subject to certain conditions.

\section{Prime Square Labeling Of A Graph:}

This section we present some of our original research work.

In this section we define a prime square dominating graph and we provide necessary examples. Also we prove that if a graph contains an odd cycle then it is not a prime square dominating graph and if graph is bipartite then it is a prime square dominating graph. We provide a minimal prime square dominating graph and explained with an example.

2.1 Definition: A Graph is Prime Square dominating graph, if the vertices of graph $G$ is labeled with positive integers and the vertices labeled with composite number $n$ is adjacent to the vertices labeled with prime numbers $p$ if and only if $n$ is divided by $p^{2}$ (were $n>p^{2}$ ).

2.2 Example: Observe the following graph whose vertex set $V=\{2,3,5,36,40,100\}$ is partitioned into two sets $\{2,3,5\}$ and $\{36,40,100\}$ is a prime squaring dominating graph.

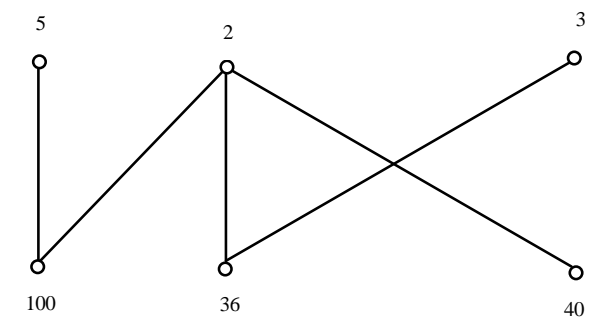

Fig - 2.2

2.3 Note: Every prime square dominating graph is a finite and simple graph.

2.4 Note: Every prime square dominating is a prime dominating but converse is not true. For example observe the following prime dominating graph.

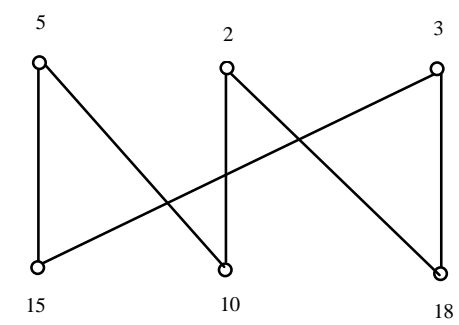

Fig-2.4

Fig-2.4 is a prime dominating graph. But it is not a prime square dominating graph.

2.5 Theorem: Any Graph containing an odd cycle is not prime square dominating graph.

Proof: Let $\mathrm{G}$ be a Graph containing an odd cycle $\mathrm{v}_{1}, \mathrm{v}_{2}, \mathrm{v}_{3}, \ldots, \mathrm{v}_{2 \mathrm{n}-1}$. If $\mathrm{G}$ has a prime square dominating then the vertices are label with prime and composite numbers alternately. Now $\mathrm{v}_{1}$ and $\mathrm{v}_{2 \mathrm{n}-1}$ were connected and both 
have same kind of label (both are primes (or) composites). This is contrary to the fact that no two primes (or) composites can be connected. Hence no Graph containing odd cycle can have a prime square dominating labeling.

2.6 Theorem: A Graph is bipartite if and only if it is a prime square dominating graph.

Proof: Let $\mathrm{G}$ be a bipartite graph. Then vertex set $\mathrm{V}$ of graph $\mathrm{G}$ can be partitioned into two sets $\mathrm{V}_{1}$ and $\mathrm{V}_{2}$ so that every edge of graph $G$ having one end vertex in $V_{1}$ and another vertex in $V_{2}$. Let $\left|V_{1}\right|=m$ and $\left|V_{2}\right|=n$. Suppose all vertices of $\mathrm{V}_{1}$ are labeled with primes and vertices of $\mathrm{V}_{2}$ labeled with composite numbers depending upon the labels in $V_{1}$. Then $\mathrm{G}$ becomes a prime square dominating graph. Conversely suppose that graph $\mathrm{G}$ is a prime square dominating graph. Then by theorem 2.5 it does not contain any odd cycle. Therefore it is a bipartite graph.

2.7 Definition: A graph is called $(\alpha, \beta)$ - prime square dominating graph if $\alpha$ is the number of composite labels and $\beta$ is the number of prime labels.

\subsection{Examples:}

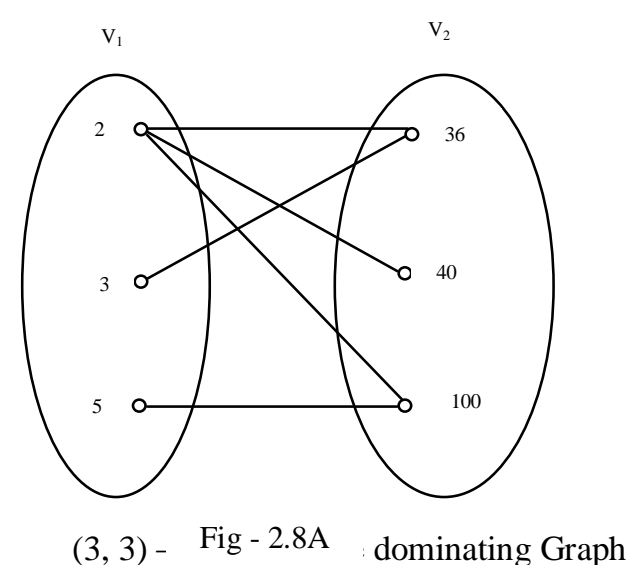

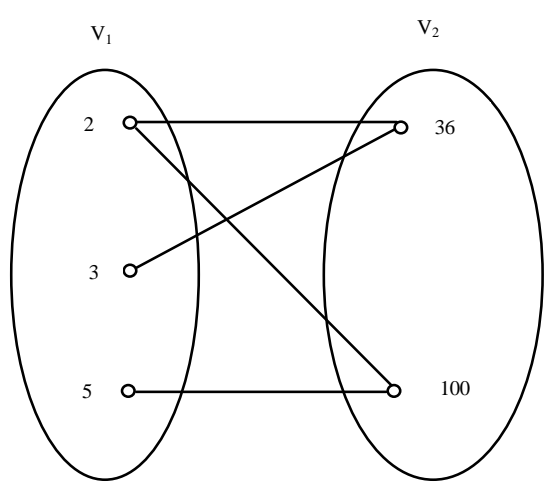

Fig - 2.8B

$(2,3)$ - Prime square dominating Graph

2.9 Definition: A Graph $G$ is called a minimal- prime square- dominating graph if $\delta(G)=\min (\alpha, \beta)$.

2.10 Example: Let $G(V, E)$ be a graph with vertex set $V=\{3,5,9,81,225\}$. Now $S=\{3,5\}$ is a dominating set and $\mathrm{N}(\mathrm{S})=\{3,5,9,81,125,225\}$. Clearly set $\mathrm{S}$ is a minimal dominating set and the cardinality of $\mathrm{S}$ is 2 . Therefore, the dominating number of the graph is 2 . Since the graph is $(4,2)$ - prime square dominating graph and $\delta(G)=\min (\alpha, \beta)=\min (4,2)=2$. Hence the following graph is a minimal prime square dominating graph.

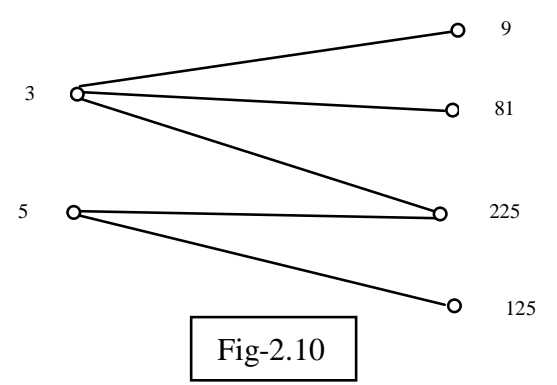

2.11 Note: Since the domination number of an $n$-cycle is equal to $\left\lfloor\frac{n}{2}\right\rfloor$ where $\lfloor x\rfloor$ is denotes the least integer greater than or equal to $x$, we can conclude that number of $2 \mathrm{n}$-cycles has a minimal prime dominating labeling. The following graph is an 8-cycle in which $\alpha=\beta=4$ and dominating number is 3 . Hence the graph is not minimal prime dominating. 


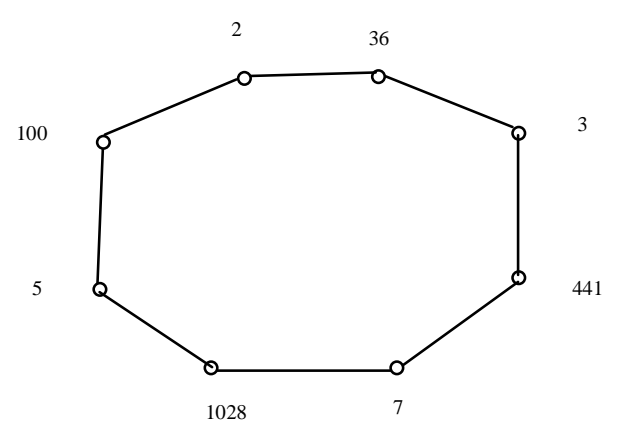

Fig-2.10

\section{Conclusion}

In our study we observed that the prime square dominating graph is not a prime dominating graph we proved that if a graph contains an odd cycle then it is not a prime square dominating graph. Moreover we observed that if graph is bipartite then it is a prime square dominating graph. Any future studies of a minimal prime square dominating graphs and also on relations between other domination parameters of a graph and prim square dominating graphs would be interesting.

\section{Acknowledgements}

Authors thanks to Prof. S.R. Sudarshan Iyengar who is the inspirer for this work. Authors also thanks to the reviewers. The second author thanks to the management of St. Mary's College of Engineering and Technology for their constant encouragement and support.

\section{References}

[1]. Harary, "Graph Theory", addition weslely 1969.

[2]. Joseph A. Gallian, "A Dynamic Survey of graph labeling", the electronic journal of combinatorics \#D S 6, 2005.

[3]. Narsing Deo "Graph Theory with Applications to Engineering and Computer Science", Prentice-Hall of India Pvt. Ltd., 1997.

[4]. S.R. Sudarshan Iyangar, Somindu C.R and Swathi Srivatsan, "Discrete Mathematics and its Applications", Narosa Publication, New Delhi, 2006. PP169-174.

[5]. Satyanarayana Bhavanari and Syam Prasad K., "Discrete Mathematics and Graph Theory", Printice Hall of India, New Delhi, 2009.

[6]. Seod M.A.and Youssef M.Z., "On prime labeling of graphs”, Congr. Numer.141(1999), 203-215.

[7]. West D. B. "Introduction to Graph Theory $-2^{\text {nd }}$ Edition", Prentice Hall of India, New Delhi, 2002. 